\title{
TriB-RT: simultaneous optimization of photon, electron and proton beams
}

To cite this article before publication: Reto Kueng et al 2020 Phys. Med. Biol. in press https://doi.org/10.1088/1361-6560/ab936f

\section{Manuscript version: Accepted Manuscript}

Accepted Manuscript is "the version of the article accepted for publication including all changes made as a result of the peer review process, and which may also include the addition to the article by IOP Publishing of a header, an article ID, a cover sheet and/or an 'Accepted

Manuscript' watermark, but excluding any other editing, typesetting or other changes made by IOP Publishing and/or its licensors"

This Accepted Manuscript is $\odot 2020$ Institute of Physics and Engineering in Medicine.

During the embargo period (the 12 month period from the publication of the Version of Record of this article), the Accepted Manuscript is fully protected by copyright and cannot be reused or reposted elsewhere.

As the Version of Record of this article is going to be / has been published on a subscription basis, this Accepted Manuscript is available for reuse under a CC BY-NC-ND 3.0 licence after the 12 month embargo period.

After the embargo period, everyone is permitted to use copy and redistribute this article for non-commercial purposes only, provided that they adhere to all the terms of the licence https://creativecommons.org/licences/by-nc-nd/3.0

Although reasonable endeavours have been taken to obtain all necessary permissions from third parties to include their copyrighted content within this article, their full citation and copyright line may not be present in this Accepted Manuscript version. Before using any content from this article, please refer to the Version of Record on IOPscience once published for full citation and copyright details, as permissions will likely be required. All third party content is fully copyright protected, unless specifically stated otherwise in the figure caption in the Version of Record.

View the article online for updates and enhancements. 


\title{
TriB-RT: Simultaneous optimization of photon,
}

\section{electron and proton beams}

\author{
R. Kueng ${ }^{1}$, S. Mueller ${ }^{1}$, H. A. Loebner ${ }^{1}$, D. Frei ${ }^{1}$, W. Volken ${ }^{1}$, \\ D. M. Aebersold ${ }^{1}$, M. F. M. Stampanoni ${ }^{2}$, M. K. Fix ${ }^{1}$, \\ P. Manser ${ }^{1}$ \\ E-mail: reto.kueng@insel.ch \\ ${ }^{1}$ Division of Medical Radiation Physics and Department of Radiation Oncology, \\ Inselspital, Bern University Hospital, and University of Bern, Switzerland \\ ${ }^{2}$ Institute for Biomedical Engineering, University of Zurich and Swiss Federal \\ Institute of Technology (ETH), Zurich, Switzerland
}

\section{Abstract.}

Purpose. To develop a novel treatment planning process (TPP) with simultaneous optimization of modulated photon, electron and proton beams for improved treatment plan quality in radiotherapy.

Methods. A framework for fluence map optimization of Monte Carlo (MC) calculated beamlet dose distributions is developed to generate treatment plans consisting of photon, electron and spot scanning proton fields. Initially, in-house intensity modulated proton therapy (IMPT) plans are compared to proton plans created by a commercial treatment planning system (TPS). A Triple Beam radiotherapy (TriBRT) plan is generated for an exemplary academic case and the dose contributions of the three particle types are investigated. To investigate the dosimetric potential, a TriB-RT plan is compared to an in-house IMPT plan for two clinically motivated cases. Benefits of TriB-RT for a fixed proton beam line with a single proton field are investigated.

Results. In-house optimized IMPT are of at least equal or better quality than TPS generated proton plans and MC-based optimization shows dosimetric advantages for inhomogeneous situations. Concerning TriB-RT, for the academic case, the resulting plan shows substantial contribution of all particle types. For the clinically motivated case, improved sparing of organs at risk close to the target volume is achieved compared 
$\operatorname{TriB}-R T$

to IMPT (e.g. myelon and brainstem $D_{\max }-37 \%$ ) at cost of an increased low dose bath (healthy tissue $V_{10 \%}+22 \%$ ). In the scenario of a fixed proton beam line, TriB-RT plans are able to compensate the loss in degrees of freedom to substantially improve plan quality compared to a single field proton plan.

Conclusion. A novel TPP which simultaneously optimizes photon, electron and proton beams was successfully developed. TriB-RT shows the potential for improved treatment quality and is especially promising for cost-effective single-room proton solutions with a fixed beamline in combination with a conventional linac delivering photon and electron fields.

Keywords: Triple Beam radiotherapy, TriB-RT, simultaneous optimization, Monte Carlo

\section{Introduction}

The fundamental goal of radiotherapy is to deliver a therapeutically efficacious dose to a target volume while limiting the exposition of normal tissue (NT). The exploitation of the so-called therapeutic window allows for good prospects of tumour control and low probability of adverse effects. Many technological advances in the past decades have paved the way to achieve this goal. Increasingly complex field arrangements, sophisticated treatment techniques, image-guidance for patient position verification and anatomy tracking have enabled steeper dose gradients at the border of the high dose region and lower doses to the NT (Schultheiss 2017). In photon radiotherapy, inverse treatment planning techniques have enabled a paradigm change towards modulated therapies such as intensity modulated radiotherapy (IMRT) and volumetric modulated arc therapy (VMAT) (Yu 1995; Bortfeld 2006; Otto 2008; Cho 2018). The enabling of both intensity and energy modulation is also promising for electron beams, which are well suited for treating superficially located targets due to the high dose at the surface/and the steep dose fall-off at larger depths (Ding et al. 1996; Hogstrom et al. 2006; Henzen et al. 2014b). The beneficial dosimetric characteristics of charged particles are even more pronounced for proton beams. Proton therapy can provide a dosimetric 
advantage over conventional photon therapy as the depth-dose characteristics of protons allow dose reduction in NT proximal and distal to the target volume (Paganetti 2016), However, due to scattering of protons in patient tissue, the penumbra of a clinical proton beam is larger than of a clinical photon beam from a certain penetration depth, which may yield higher dose to laterally situated NT (Deluca et al. 2007; Mohan et al. 2017). The combination of more than one particle type in radiotherapy is well established in clinical practice by using electron therapy subsequent to photon irradiation for postmastectomy chest wall irradiation (Kirova et al. 2007). More sophisticated approaches using photon and electron beams in mixed beam radiotherapy (MBRT) allow for ameliorated treatment quality for a wide-range of indications compared to photon or electron only therapy (Mueller et al.2017; Míguez et al. 2017; Renaud et al. 2017; Mueller et al. 2018b). Morevover, combined proton-photon treatments have recently been investigated by Unkelbach et al. 2018 under consideration of limited proton treatment slots. As an increasing number of hospitals install single-room proton therapy facilities, combining proton therapy with linear accelerator provided photon and electron beams becomes a clinical option. However, to the best of our knowledge, there is no study on the development of a treatment planning process (TPP) with simultaneous optimization of photon, electron and proton beams in radiotherapy. The purpose of this work is twofold: Firstly, an in-house solution for Monte Carlo (MC) based IMPT plan optimization is developed and compared to proton plans created by a commercial treatment planning system (TPS). The second aim is to develop Triple Beam radiotherapy/(TriB-RT), a novel TPP with simultaneous optimization of modulated photon, electron and proton beams. Fluence map optimization of MC calculated beamlet dose distributions is used to harvest the benefits of all three beam types. An academic case is used to illustrate the characteristics of TriB-RT and two clinically motivated cases are investigated by comparing TriB-RT plans with IMPT plans. 


\section{$\operatorname{TriB}-R T$}

\section{Materials and Methods}

\subsection{Treatment planning process for TriB-RT}

In the first step of the TPP for TriB-RT, the CT dataset is imported into a research version of the Eclipse TPS (Varian Medical Systems, Palo Alto, CA). The clinically relevant structures such as the planning target volume (PTV) and organs at risk (OARs) are contoured and the physician's intent for prescription dose and fractionation scheme is defined. Subsequently, photon, electron and proton beam directions including isocenter positions are manually set up. Following the principles of MBRT planning (Mueller et al. 2017), supported beam energies and collimator rotation are defined for photon and electron fields. The photon and electron plan contributions are designed to be delivered on a TrueBeam (Varian Medical Systems, Palo Alto, CA) with a source-axis distance of $100 \mathrm{~cm}$, which is equipped with a Millennium 120 pMLC (Varian Medical Systems, Palo Alto, CA). All photon and proton beams are defined in a single isocenter group around the PTV, whereas electron fields are set up/with reduced source to surface distance (SSD) of 70-85 cm to minimize air scattering (Mueller et al. 2018a). In this work, proton beam contribution is designed to be delivered using the the ProBeam ${ }^{\mathrm{TM}}$ spot scanning system (Varian Medical Systems, Palo Alto, CA) with a source-axis distance of $228 \mathrm{~cm}$ and spot candidates to cover the target are determined using the Eclipse TPS. If a proton field is not able to adequately cover the superficial part of the PTV due to the lower energy boundary, a range shifter (RS) is added as a pre-absorber to the respective proton field. Beamlet dose calculations are performed using MC simulations as described in the subsequent paragraph. For the photon and electron fields, a beamlet grid with a resolution of $0.5 \times 0.5 \mathrm{~cm}^{2}$ (at isocenter level) is positioned on the center plane of the photon multileaf collimator (pMLC). For the spot scanning proton fields, the contribution of each individual spot is considered a beamlet dose. For the simultaneous optimization process of all particle types, the beamlet dose distributions in units Gy per

MU and the dose objectives with priorities are input to the optimizer and the output is 


\section{$\operatorname{TriB}-R T$}

the absolute weight of each beamlet in MUs.

\subsection{Monte Carlo simulations}

The Eclipse TPS is interfaced with the Swiss Monte Carlo Plan (SMCP) framework (Fix et al. 2007) to initiate beamlet dose calculation. For the $6 \mathrm{MV}$ photon beamlets, a validated phase space beam model (Magaddino et al. 2011) and VMC ${ }^{++}$(Kawrakow et al. 2000) dose calculation is applied. Electron beamlets with energies 6, 9, 12, 15, 18 and $22 \mathrm{MeV}$ are calculated using a multiple source MC beam model (Henzen et al. 2014a) and patient dose calculation is done using the electron macro MC algorithm (Neuenschwander et al. 1992, 1995; Fix et al. 2013a). For proton beamlets, a generic beam model of the ProBeam ${ }^{\mathrm{TM}}$ system is used, which is commissioned for proton energies between $70-244 \mathrm{MeV}$ and includes an optional RS with a water equivalent thickness of $5.7 \mathrm{~cm}$. Dose calculation is performed with the proton macro MC algorithm (Fix et al. 2013b; Kueng et al. 2019). A voxel size of $2.5 \times 2.5 \times 2.5 \mathrm{~mm}^{3}$ is used for all dose calculations in this work. The average relative statistical uncertainty (one standard error of the mean) of the voxels with dose values higher than $50 \%$ of the maximal dose is below $1 \%$ for all presented dose distributions, as determined by a history-byhistory method (Walters et al. 2002). The statistical uncertainty in the beamlet dose distributions can propagate to variability in the subsequent optimization process (Jeraj et al. 2000). This is mitigated by ensuring the relative statistical uncertainty of each individual beamlet dose distribution to be lower than $2 \%$.

\subsection{TriB-RT and in-house IMPT optimization}

In order to optimize TriB-RT and in-house IMPT plans, dose-volume objectives (Wu et al. 2000), generalized equivalent uniform dose (gEUD) objectives (Niemierko 1999) as well as NT objectives (Varian Medical Systems 2014) are defined by the planner. The objective function to be minimized in the optimization process is defined as a weighted 


\section{$\operatorname{TriB-RT}$}

sum

$$
f=\sum_{k=1}^{N_{\mathrm{DV}}} w_{k} \cdot f_{k}^{\mathrm{DV}}+\sum_{j=1}^{N_{\mathrm{gEUD}}} w_{j} \cdot f_{j}^{\mathrm{gEUD}}+\sum_{l=1}^{N_{\mathrm{NT}}} w_{l} \cdot f_{l}^{\mathrm{NT}},
$$

where $w_{k}$ is the weight of the $k$ th of $N_{\mathrm{DV}}$ dose-volume objectives

$$
f_{k}^{\mathrm{DV}}=\sum_{i=1}^{M_{K}} \frac{\theta\left(a_{k} \cdot\left(D_{i}-D_{k}\right)\right) \cdot \theta\left(a_{k} \cdot\left(D\left(V_{k}\right)-D_{i}\right)\right) \cdot\left(D_{i}-D_{k}\right)^{2}}{N_{i}}
$$

and $w_{j}$ is the weight of the $j$ th of $N_{\mathrm{gEUD}}$ gEUD objectives

$$
f_{j}^{\mathrm{gEUD}}=\theta\left(\mathrm{gEUD}(t, j)-\mathrm{gEUD}_{j}\right) \cdot\left(\operatorname{gEUD}(t, j)-\mathrm{gEUD}_{j}\right)^{2} .
$$

In a dose-volume objective, $D_{i}$ is the dose in the $i$ th of $M_{K}$ voxels of the considered structure, $D_{k}$ is the objective dose, $D\left(V_{k}\right)$ is the dose to at least the tolerated volume $V_{k}$ and $\theta$ is the Heaviside step function. Upper and lower objectives are identified by the parameter $a_{k}= \pm 1$, respectively, For an objective $\operatorname{gEUD}_{j}, \operatorname{gEUD}(t, j)=$ $\left(\frac{1}{M_{j}} \cdot \sum_{i=1}^{M_{j}}\left(D_{i}\right)^{t}\right)^{1 / t}$ is characterized by a tissue-specific parameter $t$ and $D_{i}$ is the dose to the $i$ th of $M_{j}$ voxels of the structure of interest. NT objectives are defined by

$$
f_{l}^{\mathrm{NT}}=\frac{1}{V_{l}} \sum_{i=1}^{M_{l}} v_{i} \cdot \theta\left(D_{i}-D_{i}^{N T}\right) \cdot\left(D_{i}-D_{i}^{N T}\right)^{2},
$$

where $V_{l}$ is the volume and $M_{l}$ the number of voxels of the NT structure $l, v_{i}$ is the fractional volume overlap of the voxel $i$ with the NT structure, $D_{i}$ is the dose delivered to the voxel $i$ and $D_{i}^{N T}$ is the desired dose at voxel $i$ defined by

$$
D_{i}^{N T}=\left\{\begin{array}{cl}
f_{0} \cdot e^{-k\left(x_{i}-x_{\text {start }}\right)}+f_{\infty} \cdot\left(1-e^{-k\left(x_{i}-x_{\text {start }}\right)}\right) & \text { if } x_{i} \geq x_{\text {start }} \\
f_{0} & \text { otherwise }
\end{array},\right.
$$

where $f_{0}$ is the start dose, $f_{\infty}$ the end dose, $k$ the fall-off parameter, $x_{i}$ the distance of the voxel $i$ to the closest PTV voxel and $x_{\text {start }}$ is the start distance.

Fluence map optimization (FMO) is applied to determine the optimized MUs of each beamlet for both TriB-RT and IMPT optimization. In this work, a limited-memory Broyden-Fletcher-Goldfarb-Shanno (L-BFGS) algorithm (Broyden 1970; Nocedal et al. 1999) is applied, which belongs to the class of Quasi-Newton methods. The length of the L-BFGS history is set to 4 and the optimization continues for at most $10^{4}$ iterations or if the relative difference of the objective function value to the previous value is smaller than $10^{-8}$ for three consecutive iterations. 


\subsection{Academic case}

To emphasize the fundamental characteristics of TriB-RT, an academic situation is investigated. The academic case consists of a cubic $30 \times 30 \times 30 \mathrm{~cm}^{3}$ homogeneous water phantom with cuboid OARs and PTV, shown in figure 1 . The PTV, with a base of $10 \times 10 \mathrm{~cm}^{2}$ is located $0.5 \mathrm{~cm}$ from the surface, reaching to a depth of $14 \mathrm{~cm}$ in the water phantom. There are two cuboid OARs (base $2 \times 10 \mathrm{~cm}^{2}$ ) located at a close lateral proximity of $0.5 \mathrm{~cm}$ on opposing sides of the PTV. The two remaining sides are not covered by OARs. There is an additional cubic OAR with a base length of $5 \mathrm{~cm}$ situated on the distal part of the PTV at a distance of $0.5 \mathrm{~cm}$.

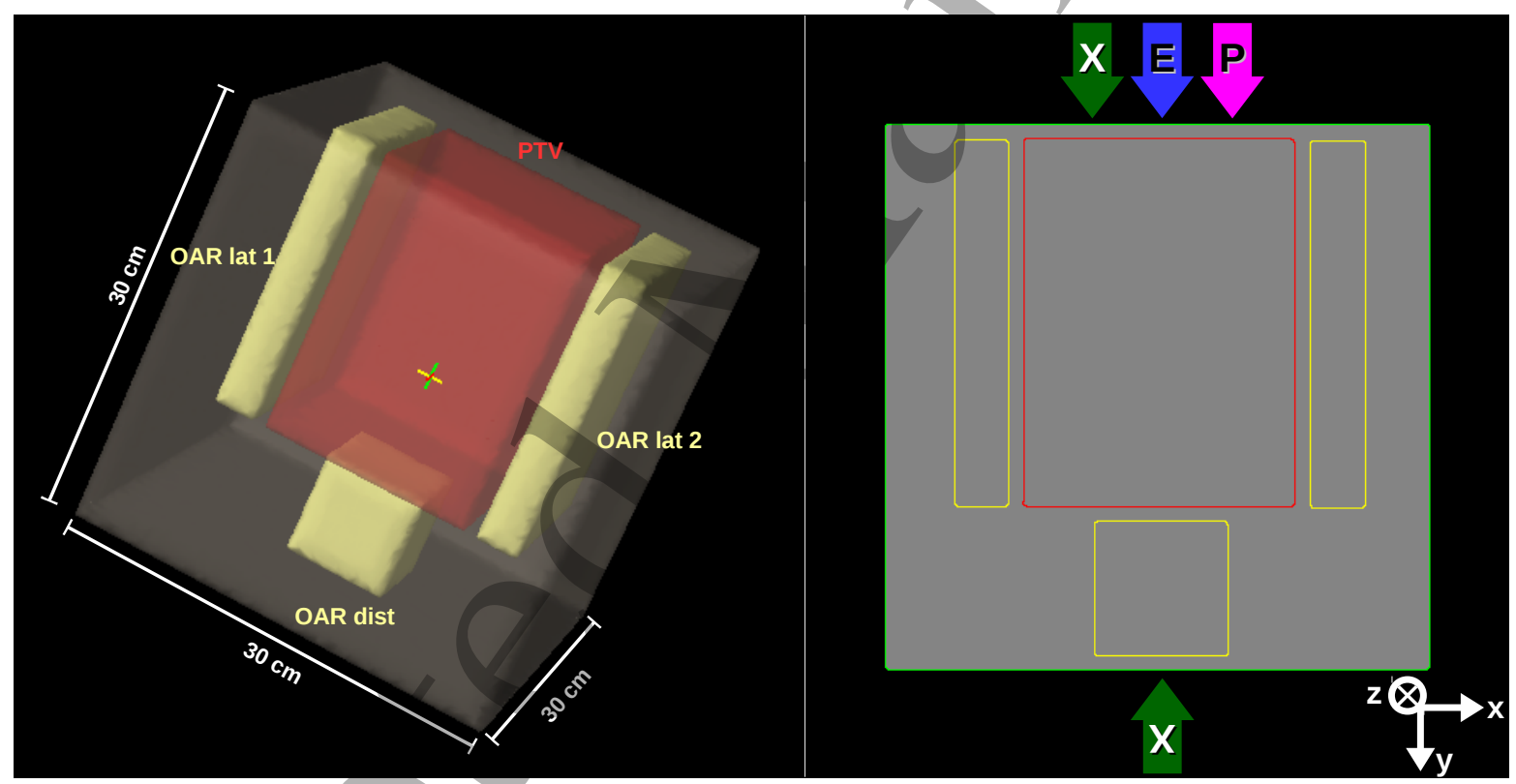

Figure 1: Left: $3 D$ view of the academic case with PTV (red) and OARs (yellow). Right: Field setup for the photon $(X)$, electron $(E)$ and proton $(P)$ fields.

For the photon fields, two field directions at gantry angle $0^{\circ}$ and $180^{\circ}$ are defined. Electron fields and a spot scanning proton field are set up from a single direction at gantry angle $0^{\circ}$.

An arbitrary dose of 100 Gy is prescribed to the median dose of the PTV. Upper and lower dose-volume objectives are set on the PTV to ensure homogeneous coverage and maximum dose objectives $D_{\max }=0$ Gy are defined for the three OARs. 
$\operatorname{TriB}-R T$

\subsection{Head and neck case}

The first clinically motivated case is a head and neck patient with a mostly superficial PTV which is covered by a bolus. The structures and field setup of the head and neck case are shown in figure 2. For this case, a coplanar setup is investigated and the parameters for the photon, electron and proton fields are indicated in table 1 .

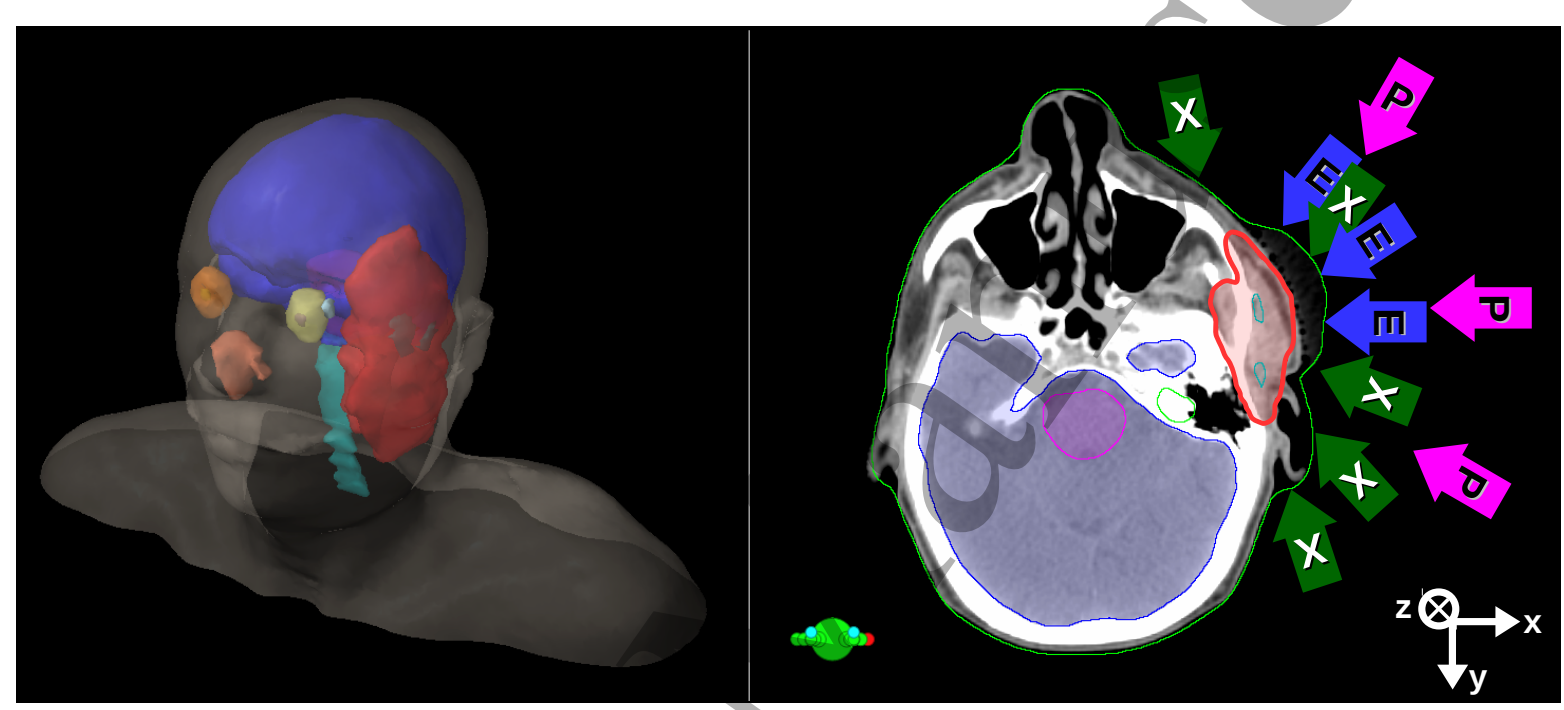

Figure 2: Left: $3 D$ view of the head and neck case with PTV and OARs. Right: Field setup for the photon, electron and proton fields.

Table 1: Coplanar setup of the photon fields (isocentric), electron fields (3 isocenters) and spot scanning proton fields (isocentric) for the head and neck case.

\begin{tabular}{|c|c|c|}
\hline Gantry $\left[{ }^{\circ}\right]$ & Energy & $\mathrm{SSD}[\mathrm{cm}]$ \\
\hline \multicolumn{3}{|c|}{ Photon fields } \\
\hline 35 & $6 \mathrm{MV}$ & 95.9 \\
\hline 111 & $6 \mathrm{MV}$ & 96.5 \\
\hline 138 & $6 \mathrm{MV}$ & 95.6 \\
\hline 162 & $6 \mathrm{MV}$ & 93.2 \\
\hline 348.5 & $6 \mathrm{MV}$ & 94.5 \\
\hline \multicolumn{3}{|c|}{ Electron fields } \\
\hline 38.5 & $6,9,12,15,18,22 \mathrm{MeV}$ & 71.0 \\
\hline 57 & $6,9,12,15,18,22 \mathrm{MeV}$ & 76.6 \\
\hline 90 & $6,9,12,15,18,22 \mathrm{MeV}$ & 80.1 \\
\hline \multicolumn{3}{|c|}{ Proton fields } \\
\hline 30 & 88.1-147.6 MeV (18 layers) with RS & 223.3 \\
\hline 90 & 87.6-143.6 MeV (17 layers) with RS & 223.9 \\
\hline 120 & 88.9-144.9 MeV (17 layers) with RS & 224.1 \\
\hline
\end{tabular}


A prescription dose of $66 \mathrm{~Gy}$ to be delivered in 33 fractions of $2 \mathrm{~Gy}$ is assigned to the median dose of the PTV. Clinically motivated optimization objectives focus on homogeneous coverage of the PTV and sparing of OARs, with focus on maximum dosevolume objectives for serial organs and mean dose objectives (gEUD with $t=1$ ) on parallel organs. An additional NT objective is set on the healthy tissue for improved dose conformity.

\subsection{Pelvic case}

The second clinically motivated case is a lymphatic tumor in the pelvic region. The PTV is surrounded by various OARs such as bowels, rectum, bladder and femoral heads. The structures and field setup of the pelvic case are shown in figure 3 .

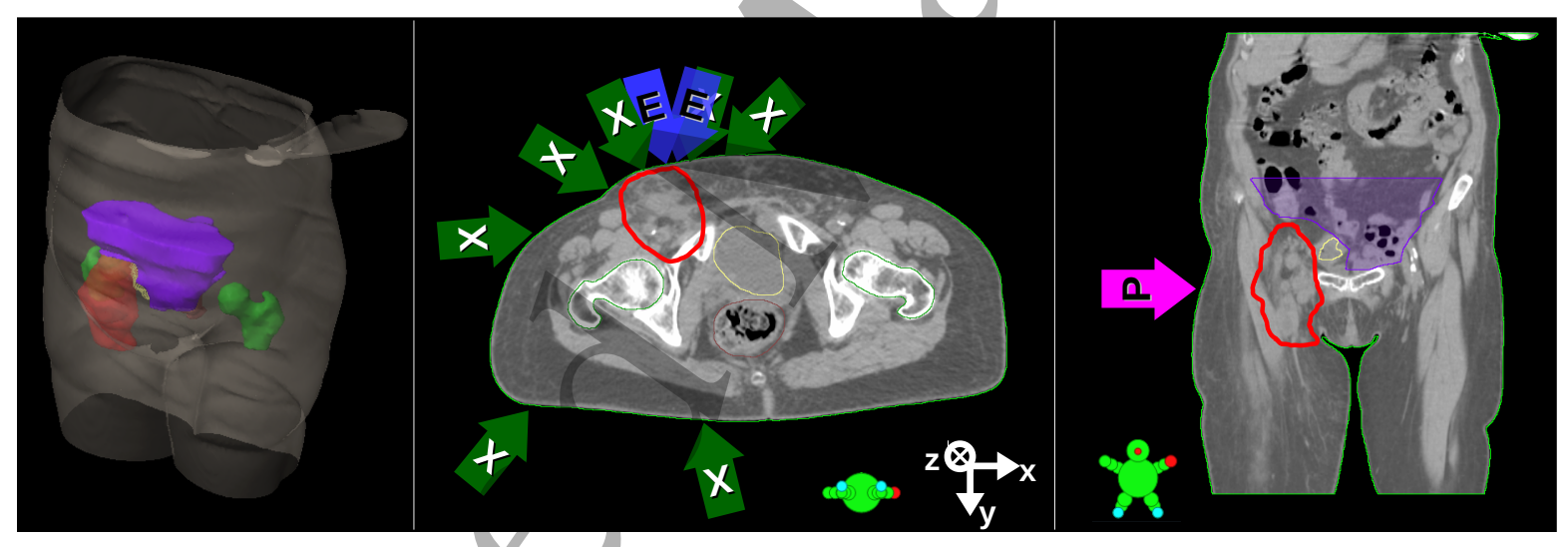

Figure 3: Left: 3D view of the pelvic case with PTV and OARs. Center: Field setup for the photon and electron fields. Right: Proton field in coronal view.

For this case, single proton field at a fixed proton gantry angle of $270^{\circ}$ is defined, again emulating the scenario of a fixed horizontal beamline proton facility. Photon and electron fields are set up in a coplanar manner around the target volume. The corresponding field parameters are indicated in table 2. A prescription dose of 50.4 Gy to be delivered in 28 fractions of $1.8 \mathrm{~Gy}$ is assigned to the median dose of the PTV. Clinically motivated optimization objectives focus on homogeneous coverage of the PTV and sparing of OARs, with focus on maximum dose-volume objectives for serial organs and mean dose objectives (gEUD with $t=1$ ) on parallel organs. An additional NT 


\section{$\operatorname{Tr} i B-R T$}

objective is set on the healthy tissue for improved dose conformity.

Table 2: Setup of the photon fields, electron fields and spot scanning proton field for the pelvic case. A coplanar setup is chosen for the photon and electron fields and a fixed horizontal proton beamline is assumed.

\begin{tabular}{|c|c|c|}
\hline Gantry $\left[^{\circ}\right]$ & Energy & $\mathrm{SSD}[\mathrm{cm}]$ \\
\hline \multicolumn{3}{|c|}{ Photon fields } \\
\hline 16 & $6 \mathrm{MV}$ & 95.7 \\
\hline 46 & $6 \mathrm{MV}$ & 93.3 \\
\hline 166 & $6 \mathrm{MV}$ & 83.8 \\
\hline 219 & $6 \mathrm{MV}$ & 81.4 \\
\hline 265 & $6 \mathrm{MV}$ & 88.7 \\
\hline 301 & $6 \mathrm{MV}$ & 95.7 \\
\hline 334 & $6 \mathrm{MV}$ & 97.3 \\
\hline \multicolumn{3}{|c|}{ Electron fields } \\
\hline 16 & $6,9,12,15,18,22 \mathrm{MeV}$ & 75.1 \\
\hline 344.5 & $6,9,12,15,18,22 \mathrm{MeV}$ & 70.1 \\
\hline \multicolumn{3}{|c|}{ Proton field } \\
\hline 270 & 86.8-177.8 MeV (27 layers) w/ RS & 218.8 \\
\hline
\end{tabular}

\subsection{Analysis}

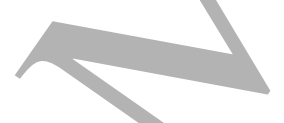

In-house vs. TPS IMPT: The plan quality of the new TriB-RT TPP will be compared to in-house optimized IMPT plans. Therefore, in order to first assess the quality of in-house optimized IMPT plans, a plan comparison between an in-house IMPT plan and a corresponding multi-field optimized (MFO) proton plan generated in the Eclipse TPS is performed. The body structure of the clinically motivated head and neck case is assigned a fixed Hounsfield number 0, therefore masking all tissue inhomogeneities. This serves the purpose of eliminating potential differences in the underlying dose calculation algorithms for the two optimization approaches, under the premise that the TPS convolution superposition algorithm and the pMMC dose calculation are equivalent in water. The resulting TPS plan is re-calculated using pMMC and compared to the in-house optimized IMPT plan by means of DVHs and dose distributions.

The same investigation is carried out for the clinically motivated head and neck case 
for the CT set including tissue heterogeneities. Again, the resulting TPS plan is recalculated using pMMC and compared to the in-house optimized IMPT plan by means of DVHs and dose distributions.

TriB-RT proof of concept: As a proof of concept for the newly introduced TPP, a TriB-RT plan is generated for the academic case and is investigated for dose contributions of the individual particle types to the target volume.

TriB-RT vs. IMPT: Next, for the clinically motivated head and neck case, TriBRT is compared to an three-field in-house IMPT plan. The TriB-RT/plan is investigated for dose contributions of the individual particle types. Plan quality is compared between the TriB-RT and a three-field IMPT plan by means of DVH, PTV dose homogeneity and OAR dose-volume parameters.

TriB-RT vs. IMPT (single fixed proton beam): As a final investigation, a fixed proton beam line with a single horizontal beam direction is emulated, as it could be present at a single-room low cost proton facility. For both the clinically motivated head and neck and plevic case, a single field proton plan $(1 \mathrm{~F}-\mathrm{PT})$ is generated and compared to a TriB-RT (1P) plan using the same single proton field, but still allowing all introduced photon and electron beam directions. Again, plan quality is compared between the TriB-RT (1P) and 1F-PT plan by means of DVHs, PTV homogeneity and OAR dose-volume parameters.

TriB-RT vs. VMAT: For the plevic case, the resulting TriB-RT (1P) and 1F-PT plan are additionally compared to the clinically applied VMAT plan (4 co-planar half arcs around the right body side). 


\section{Results}

\subsection{IMPT optimization}

For the clinically motivated head and neck case, the plan comparison between an MFO proton plan generated in the TPS and in-house optimized IMPT plan is presented in Appendix A for the water equivalent scenario. The corresponding dose distributions for a representative slice of the planning CT and the resulting DVHs are shown in figure A1. The MC re-calculation of the TPS optimized plan shows dose differences of up to $-5 \%$ compared to the analytical TPS dose calculation algorithm. These differences are predominantly in areas of steep dose gradients, where distance-to-agreement is $<1 \mathrm{~mm}$. This demonstrates that for a water equivalent situation, the TPS analytical dose calculation algorithm and the pMMC algorithm are equivalent. Comparing the in-house optimized IMPT plan to the re-calculated TPS proton plan, it can be observed that the in-house optimized IMPT/plan shows a more conformal dose distribution to the target volume. Local dose differences of up to $-25 \%$ of the prescription dose (corresponds to up to -16.5 Gy over the entire course of treatment) can be observed in the NT area enclosed by the target volume. Considering the DHVs, only small differences can be observed between the two plans.

The results of the analogue investigation for the clinically motivated head and neck case including tissue inhomogenities are shown in figure 4. Larger differences between the re-calculated and the original TPS plan can be observed in this case, with differences of up to $-20 \%$. This propagates to differences in the DVHs, where a degradation of the PTV DVH can be observed for the re-calculated plan compared to the original TPS plan. The in-house optimized IMPT plan in contrast is optimized based on MC calculated beamlet dose distribution and thus does not suffer from degradation between optimized and re-calculated plan. The resulting plan achieves excellent conformity and dose homogeneity in the target volume. 

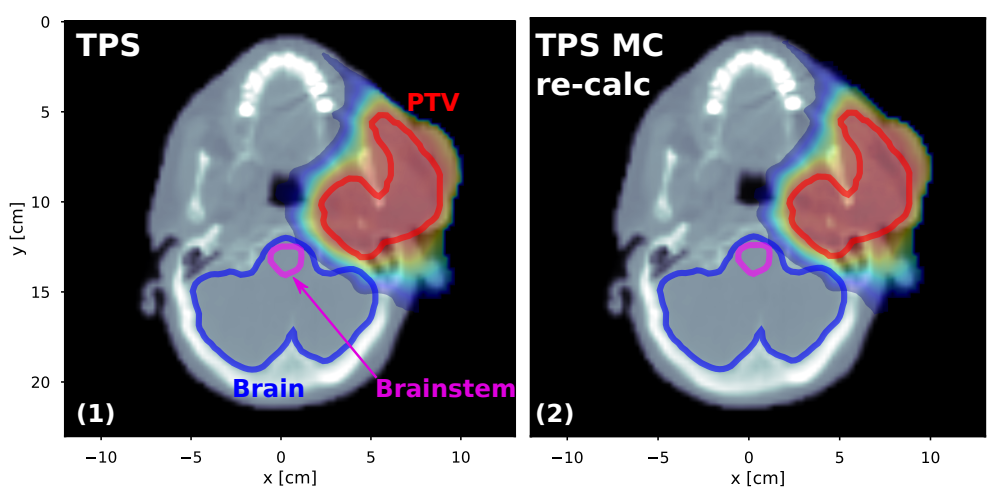

\section{in-house} IMPT
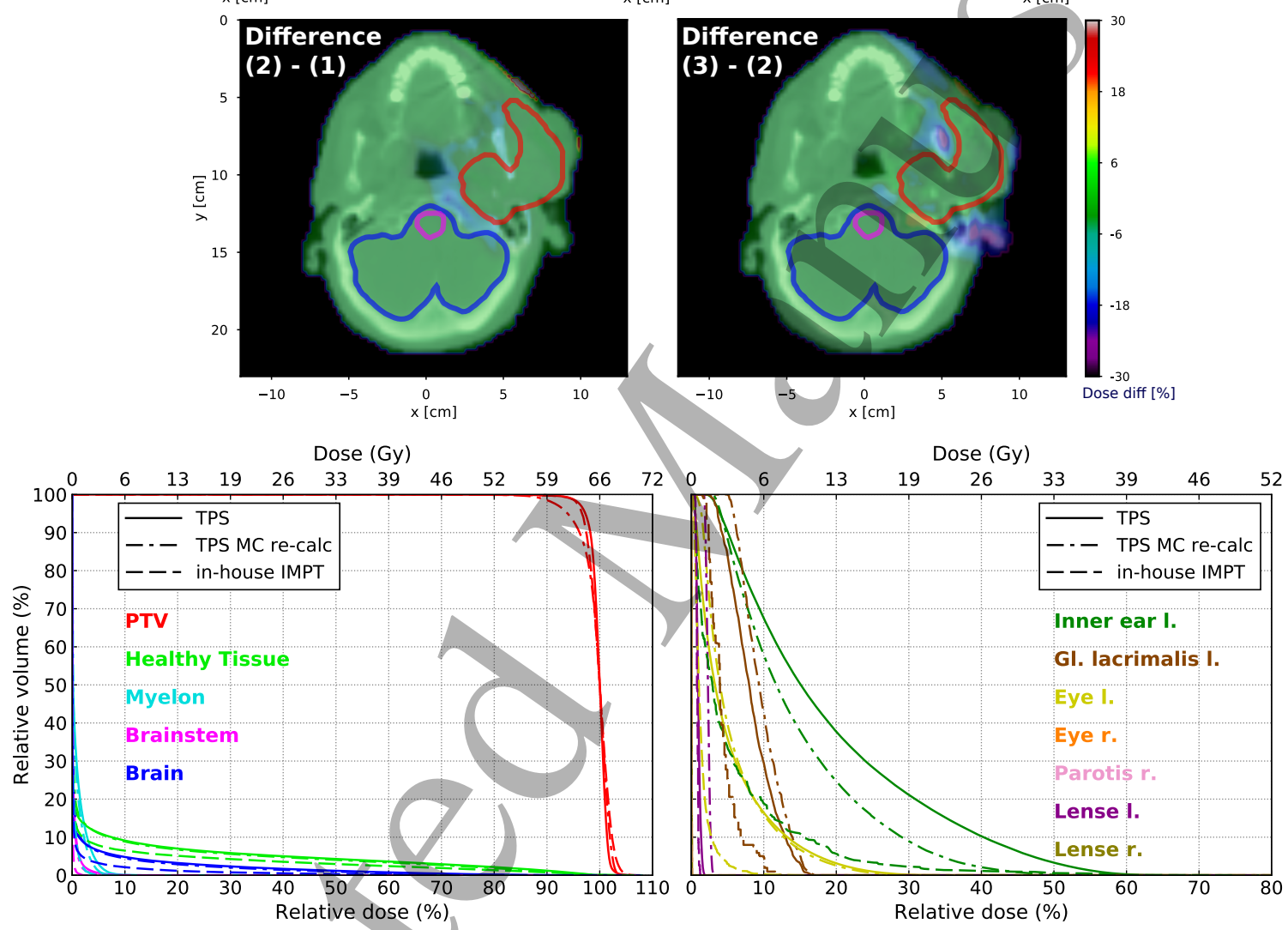

Figure 4: Top: Dose distributions of the TPS optimized proton plan (left), the same plan recalculated with $M C$ (center), and the in-house optimized IMPT plan (right) for a representative slice of the planning CT, with PTV contour indicated in red. Middle: Relative dose difference (\% of prescription dose) between the re-calculated and original TPS plan (left) and between in-house IMPT and MC re-calculated TPS plan (right). Bottom: DVHs comparing the resulting TPS optimized proton plan (solid), re-calculated TPS plan (dash-dot) and in-house optimized IMPT plan (dashed) for the head and neck case. 


\subsection{TriB-RT: Academic case}

As a proof of concept, a TriB-RT plan is generated for the academic case and the resulting dose contributions are shown in figure 5 .
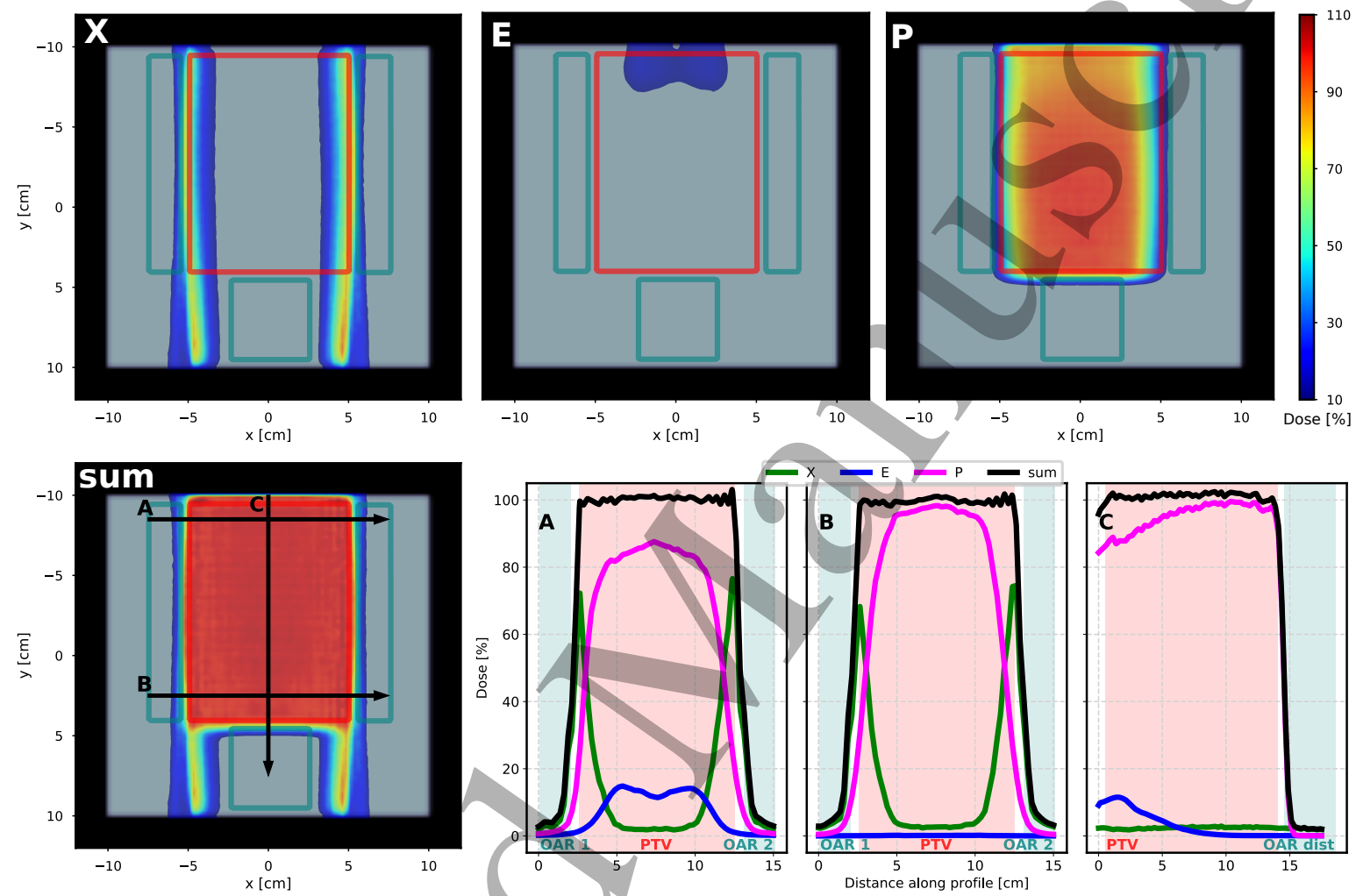

Figure 5: Top: Dose contributions by photon, electron and proton beams to the resulting TriB-RT plan for the academic case. Bottom: Total dose distribution and profiles indicating the respective contributions of the three particle types and corresponding dose profiles along the indicated arrows.

The spatial dose distributions show that the photon fields contribute mainly to the lateral boundaries of the target volume, where OARs are close-by. As expected, electron contribution can only be observed in the superficial part of the target volume. The proton field contributes dose to almost the entire target volume. The contribution to the mean dose in the PTV are $21.7 \%, 4.2 \%$ and $74.1 \%$ for the photon, electron and proton beams, respectively. Considering the total dose distribution, a very homogeneous and conformal coverage of the target volume can be observed. 


\subsection{TriB-RT: Head and neck case}

A TriB-RT plan is compared to our in-house optimized IMPT plan for the clinically motivated head and neck case and the resulting DVHs are shown in figure 6.
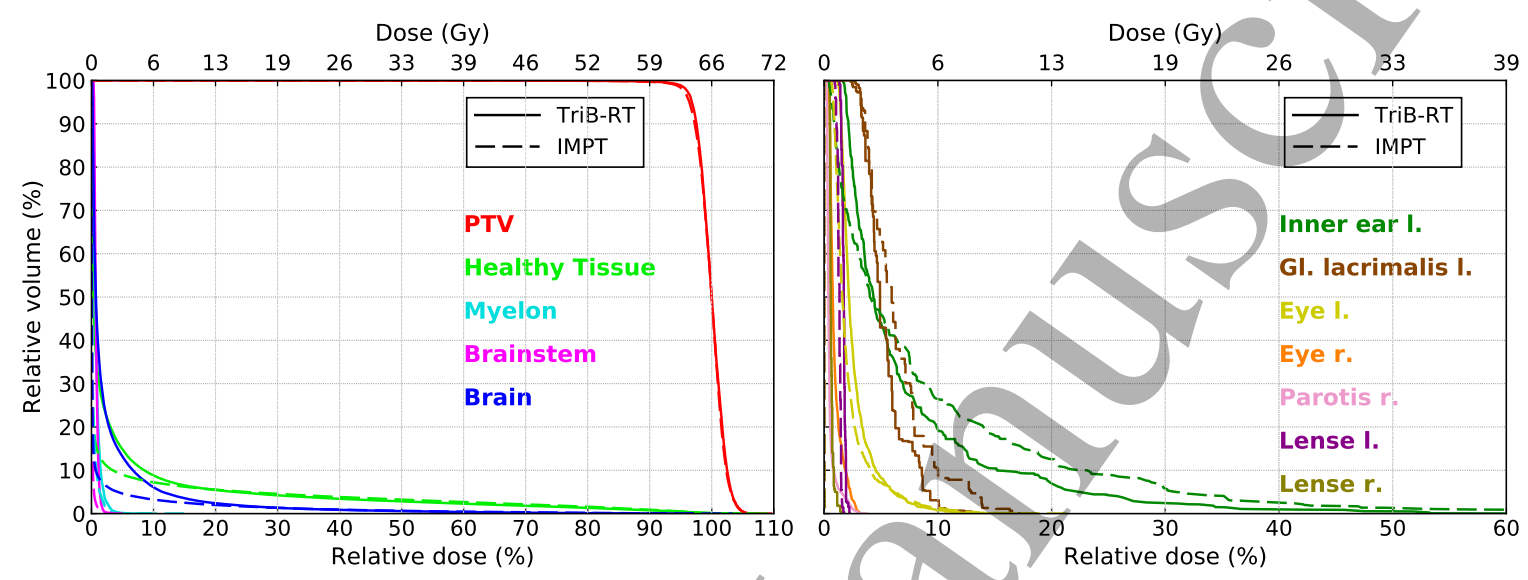

Figure 6: DVHs comparing the resulting TriB-RT (solid) and IMPT (dashed) plan for the head and neck case.

Very similar PTV dose homogeneity is achieved for both the TriB-RT and the IMPT plan with a homogeneity index $H I:=V_{95 \%}-V_{107 \%}$ of $98.9 \%$ and $98.3 \%$, respectively. Considering the OARs, TriB-RT shows improved dosimetric parameters for all organs except for the contralaterally located OARs and the ipsilateral lens, as shown in table 3. For this clinically motivated case, IMPT is beneficial in reducing the volume of the dose bath to the healthy tissue $V_{10 \%}$ (volume receiving $10 \%$ of the prescribed dose or more), which is $490 \mathrm{~cm}^{3}$ for IMPT compared to $599 \mathrm{~cm}^{3}$ for TriB-RT. However, no substantial differences in $V_{20 \%}$ can be observed. The dose contributions of the generated TriB-RT plan are shown in figure 7 for a representative slice of the patient CT. It can be observed that the photon dose contribution is most prominent in the boundary region of the target volume. The electron fields contribute minorly to the superficial part of the PTV, while the contribution of the proton fields is dominating in the corpus of the target volume. The contribution to the mean dose in the PTV are 10.0\%, $4.3 \%$ and $85.7 \%$ for the photon, electron and proton beams, respectively. This result suggests that for this case, a 3-field IMPT plan yields an excellent treatment plan, but small 
Table 3: Dosimetric parameters for the clinically motivated head and neck case. TriB-RT and IMPT are compared for the case of 3 proton fields (2nd and 3rd column) and one single proton field (4th and 5th column), superior values are indicated in bold.

\begin{tabular}{|c|c|c|c|c|}
\hline Plan & IMPT $(3 \mathrm{P})$ & TriB-RT (3P) & $1 \mathrm{~F}-\mathrm{PT}$ & TriB-RT (1P) \\
\hline PTV - HI & $98.3 \%$ & $98.9 \%$ & $93.9 \%$ & $98.7 \%$ \\
\hline Inner ear l. - $D_{\text {mean }}$ & $5.79 \mathrm{~Gy}$ & $4.77 \mathrm{~Gy}$ & $27.15 \mathrm{~Gy}$ & $7.73 \mathrm{~Gy}$ \\
\hline Lacrimal gland 1. - $D_{\text {mean }}$ & $4.26 \mathrm{~Gy}$ & $3.56 \mathrm{~Gy}$ & 24.79 Gy & $4.33 \mathrm{~Gy}$ \\
\hline Brain - $D_{1 \%}$ & $24.78 \mathrm{~Gy}$ & $23.87 \mathrm{~Gy}$ & $44.62 \mathrm{~Gy}$ & $38.56 \mathrm{~Gy}$ \\
\hline Brainstem - $D_{\max }$ & $3.52 \mathrm{~Gy}$ & $2.21 \mathrm{~Gy}$ & $22.59 \mathrm{~Gy}$ & $10.03 \mathrm{~Gy}$ \\
\hline Myelon - $D_{\max }$ & $9.86 \mathrm{~Gy}$ & $6.28 \mathrm{~Gy}$ & $35.72 \mathrm{~Gy}$ & 22.57 Gy \\
\hline Eye 1. - $D_{\max }$ & $14.39 \mathrm{~Gy}$ & $11.74 \mathrm{~Gy}$ & $38.95 \mathrm{~Gy}$ & $14.46 \mathrm{~Gy}$ \\
\hline Eye r. - $D_{\max }$ & 0.07 Gy & $2.13 \mathrm{~Gy}$ & 0.04 Gy & $2.46 \mathrm{~Gy}$ \\
\hline Lens l. - $D_{\max }$ & $1.17 \mathrm{~Gy}$ & $1.47 \mathrm{~Gy}$ & $19.51 \mathrm{~Gy}$ & 2.7 Gy \\
\hline Lens r. $D_{\max }$ & $0.04 \mathrm{~Gy}$ & $1 \mathrm{~Gy}$ & $0.03 \mathrm{~Gy}$ & $1.16 \mathrm{~Gy}$ \\
\hline Parotid gland r. - $D_{\text {mean }}$ & $0.01 \mathrm{~Gy}$ & $0.33 \mathrm{~Gy}$ & $0.01 \mathrm{~Gy}$ & $0.64 \mathrm{~Gy}$ \\
\hline Healthy Tissue - $V_{10 \%}$ & $490 \mathrm{~cm}^{3}$ & $599 \mathrm{~cm}^{3}$ & $655 \mathrm{~cm}^{3}$ & $849 \mathrm{~cm}^{3}$ \\
\hline Healthy Tissue - $V_{20 \%}$ & $375 \mathrm{~cm}^{3}$ & $369 \mathrm{~cm}^{3}$ & $509 \mathrm{~cm}^{3}$ & $493 \mathrm{~cm}^{3}$ \\
\hline 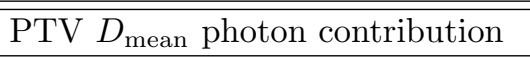 & - & $10.0 \%$ & - & $18.2 \%$ \\
\hline PTV $D_{\text {mean }}$ electron contribution & - & $4.3 \%$ & - & $26.9 \%$ \\
\hline PTV $D_{\text {mean }}$ proton contribution & $100 \%$ & $85.7 \%$ & $100 \%$ & $54.9 \%$ \\
\hline
\end{tabular}

improvements can be achieved allowing additional photon and electron beams.

However, plan quality of a pure proton plan is compromised when only a single beam direction is available. This is illustrated by the DVHs in figure 8, where a TriB-RT plan using a single proton field (1P) is compared to a single field proton plan (1F-PT). The proton field is applied at a gantry angle of $90^{\circ}$, emulating a fixed proton beam line. The DVHs indicate that the compromised plan quality of the $1 \mathrm{~F}-\mathrm{PT}$ plan can be improved by introducing photon and electron fields in the plan optimization process. This is confirmed when investigating the dosimetric parameters as listed in table 3 . With exception of the contralateral OARs and the dose bath $V_{10 \%}$, TriB-RT (1P) outperforms the 1F-PT plan. It can be observed that the PTV homogeneity and the dosimetric parameters of numerous OARs (e.g. inner ear, lacrimal gland, ipsilateral eye) can be restored to the values of the 3-field IMPT plan by allowing photon and electron beams in addition to the single proton field. Again, the dose contributions of the generated TriB-RT plan are shown in figure 9 for a representative slice of the patient CT.

For this TriB-RT plan, the contribution to the mean dose in the PTV are $18.2 \%, 26.9 \%$ 

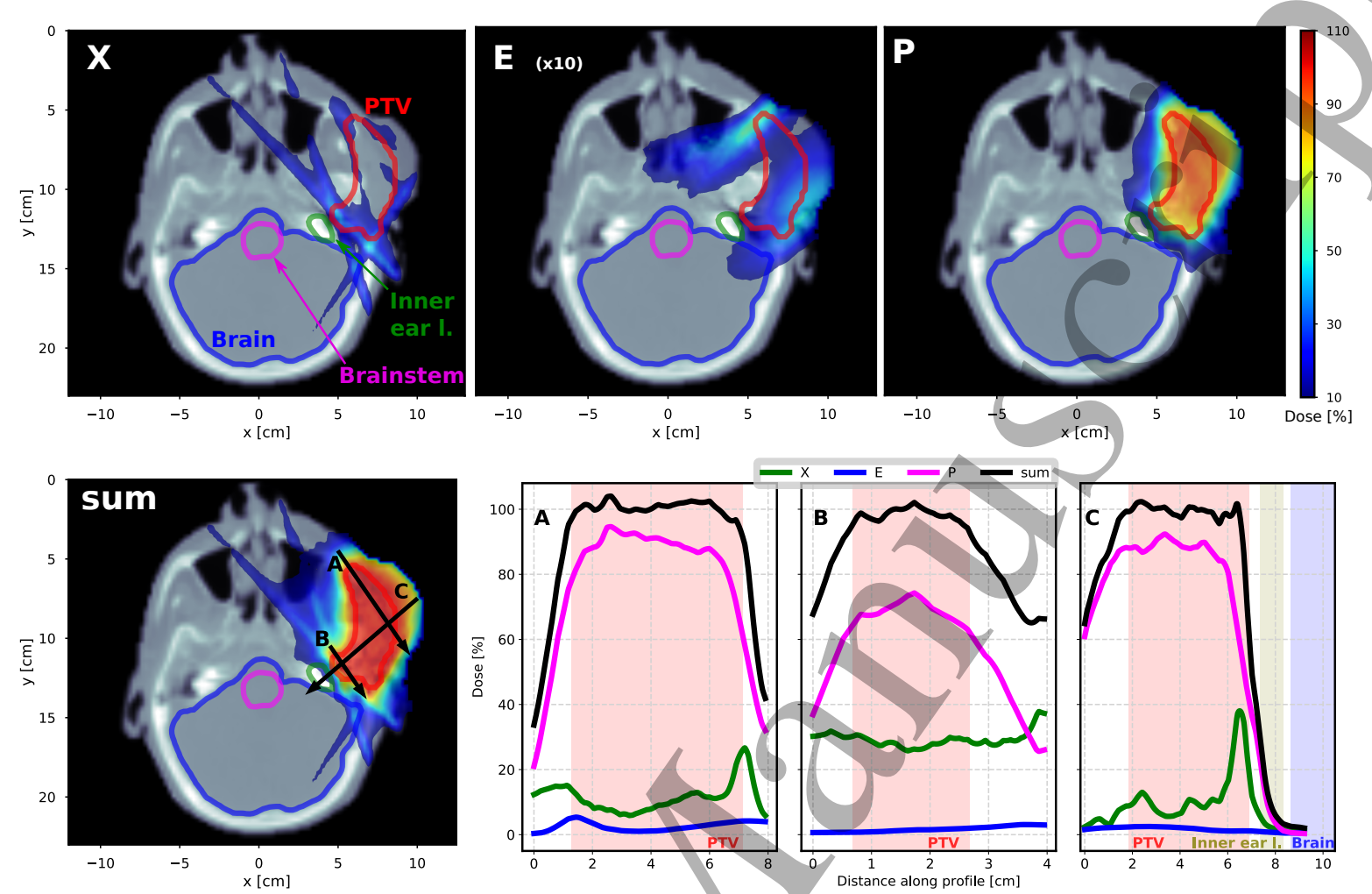

Figure 7: Top: Dose distributions by photon, electron (dose scaled by a factor of 10 for visibility) and proton beams to the resulting TriB-RT plan for the head and neck case. Bottom: Total dose distribution and profiles indicating the respective contributions of the three particle types and corresponding dose profiles along the indicated arrows.

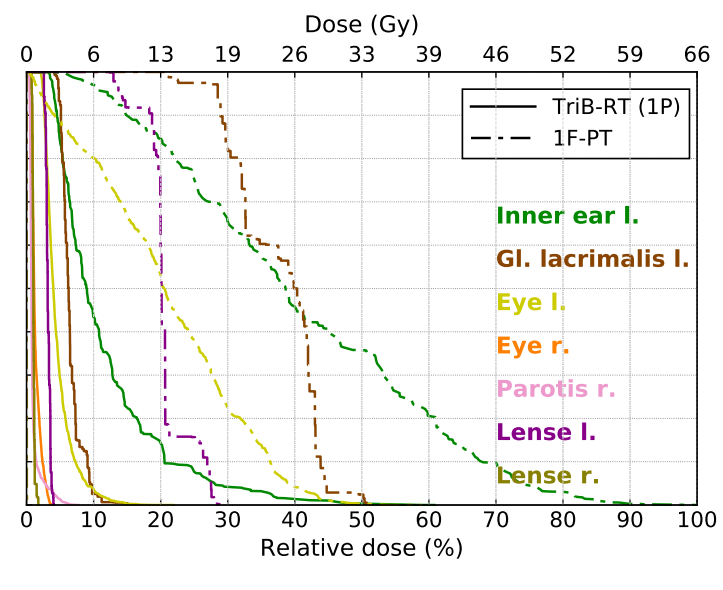

Figure 8: DVHs comparing the resulting TriB-RT (1P) (solid) and 1F-PT (dash-dotted) plan for the head and neck case. 

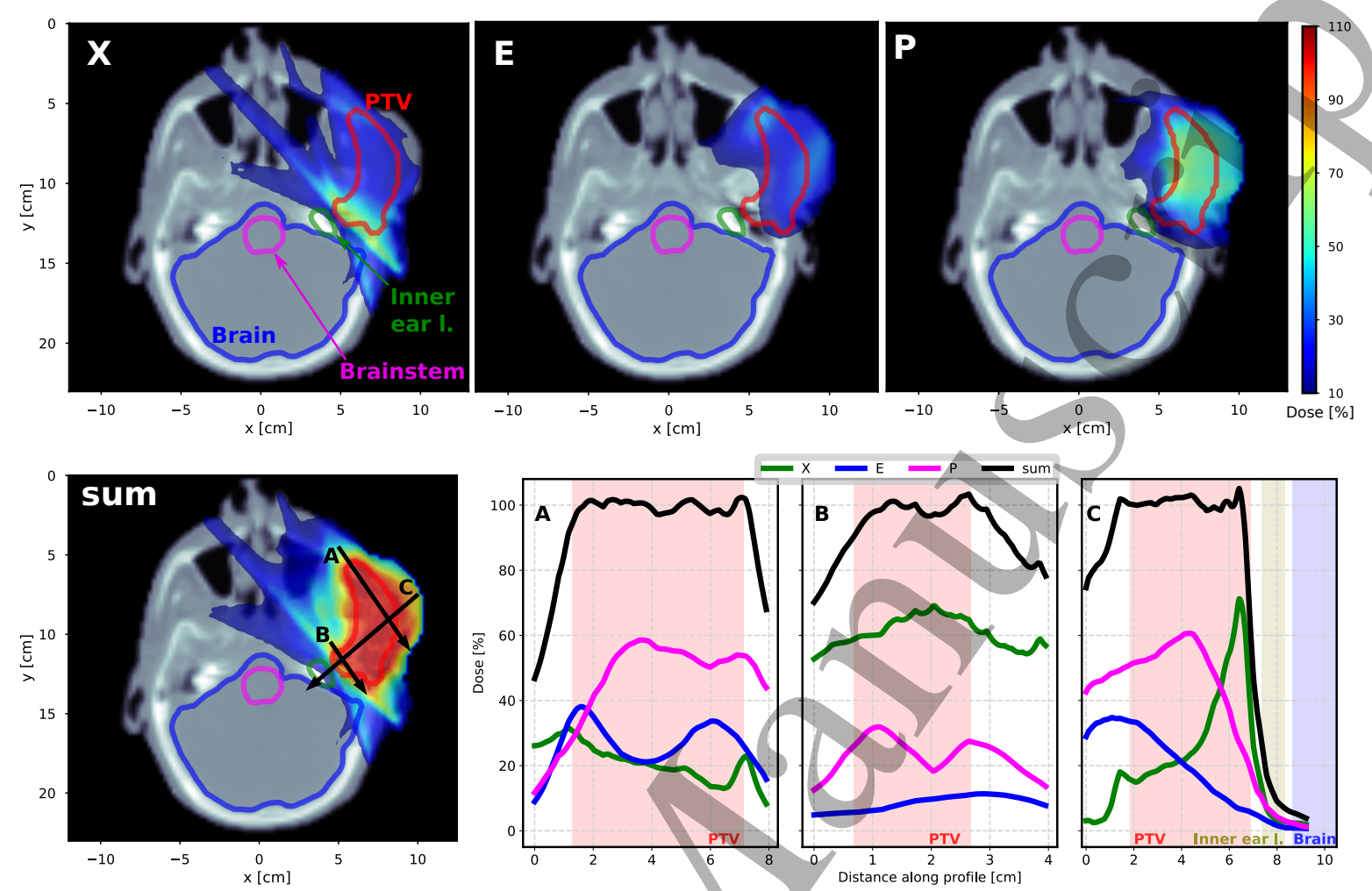

Figure 9: Top: Dose distributions by photon, electron and proton beams to the resulting TriB-RT plan for the head and neck case with only one proton field direction (90 ) available. Bottom: Total dose distribution and profiles indicating the respective contributions of the three particle types and corresponding dose profiles along the indicated arrows.

and $54.9 \%$ for the photon, electron and proton beams, respectively. Compared to the TriB-RT plan with three proton fields, the contribution of both photon and electron beams is substantially increased, showing the potential of these particles to ameliorate PTV dose homogeneity and conformity and sparing of OARs.

\subsection{TriB-RT: Pelvic case}

For the clinically motivated pelvic case, a plan comparison between a TriB-RT plan (one proton field), a single field proton plan (1F-PT), and the clinically applied VMAT plan (4 half arcs) is carried out. The corresponding DVHs are indicated in figure 10. As supported by the dosimetric values in table 4, both the VMAT and the TriB-RT plan achieve excellent PTV homogeneity of $99.8 \%$ and $99.4 \%$, respectively. The VMAT plan shows substantially larger dose to all OARs and increased low dose bath compared 
Dose (Gy)
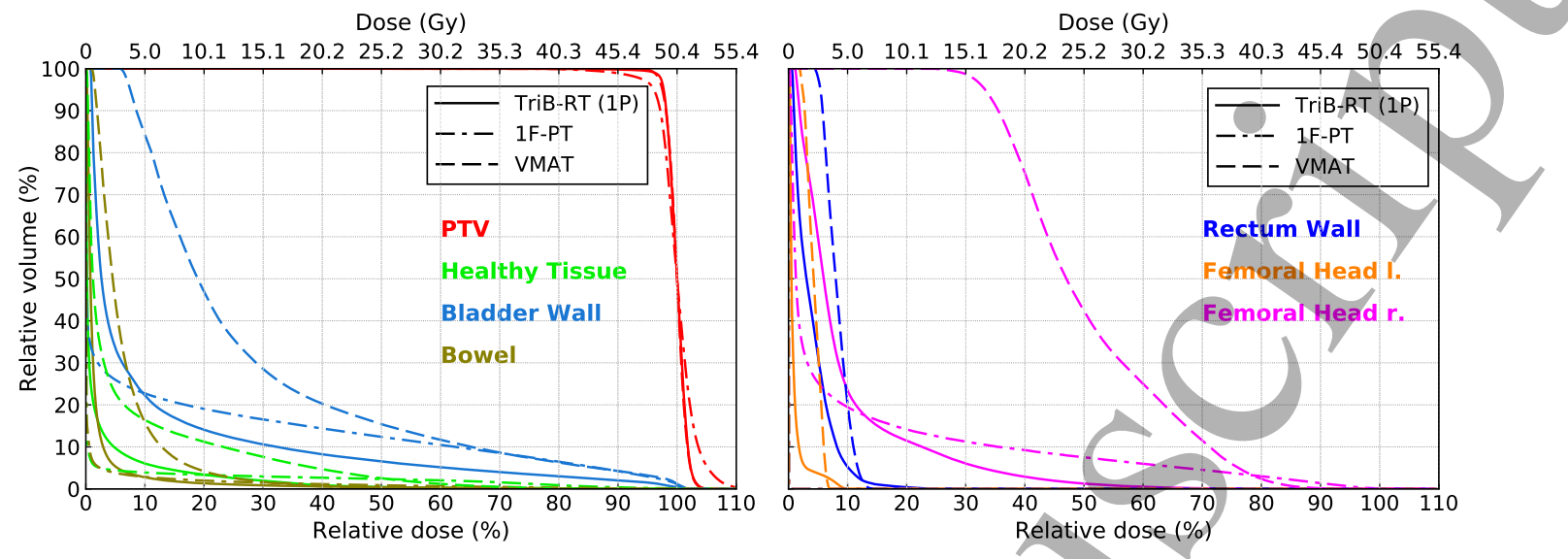

Figure 10: DVHs comparing the resulting TriB-RT (1P) (solid), $1 F-P T$ (dash-dotted) and clinical VMAT (dashed) plan for the for the pelvic case.

to the 1F-PT and the TriB-RT plan. While the low dose bath $V_{10 \%}$ is larger for the

Table 4: Dosimetric parameters for the clinically motivated pelvic case. The clinically applied VMAT plan is compared to a single field proton plan and TriB-RT plan, superior values are indicated in bold.

\begin{tabular}{|l|r|r|r|}
\hline Plan & VMAT & \multicolumn{1}{c|}{ 1F-PT } & TriB-RT (1P) \\
\hline \hline PTV - HI & $\mathbf{9 9 . 8 \%}$ & $96.7 \%$ & $99.4 \%$ \\
\hline Bladder Wall - $D_{\text {mean }}$ & $14.01 \mathrm{~Gy}$ & $6.65 \mathrm{~Gy}$ & $\mathbf{5 . 4 4} \mathbf{G y}$ \\
\hline Bowel - $D_{\text {mean }}$ & $3.28 \mathrm{~Gy}$ & $\mathbf{0 . 6 6} \mathbf{~ G y}$ & $0.96 \mathrm{~Gy}$ \\
\hline Rectum Wall - $D_{\operatorname{mean}}$ & $4.13 \mathrm{~Gy}$ & $\mathbf{0 . 0 0} \mathbf{~ G y}$ & $2.02 \mathrm{~Gy}$ \\
\hline Femoral Head r. - $D_{\max }$ & $47.12 \mathrm{~Gy}$ & $52.62 \mathrm{~Gy}$ & $\mathbf{4 0 . 9 8} \mathbf{G y}$ \\
\hline Femoral Head l. - $D_{\max }$ & $3.78 \mathrm{~Gy}$ & $\mathbf{0 . 0 5} \mathbf{~ G y}$ & $5.09 \mathrm{~Gy}$ \\
\hline Healthy Tissue- $-V_{10 \%}$ & $4891 \mathrm{~cm}^{3}$ & $\mathbf{1 1 6 2} \mathbf{c m}^{\mathbf{3}}$ & $1805 \mathrm{~cm}^{3}$ \\
\hline Healthy Tissue - $V_{20 \%}$ & $3344 \mathrm{~cm}^{3}$ & $\mathbf{9 9 7} \mathbf{c m}^{\mathbf{3}}$ & $1014 \mathrm{~cm}^{3}$ \\
\hline \hline PTV $D_{\text {mean }}$ photon contribution & $100 \%$ & - & $18.8 \%$ \\
\hline PTV $D_{\text {mean }}$ electron contribution & - & - & $47.9 \%$ \\
\hline PTV $D_{\text {mean }}$ proton contribution & - & $100 \%$ & $33.3 \%$ \\
\hline
\end{tabular}

TriB-RT plan compared to the proton plan, no substantial differences are seen in $V_{20 \%}$. Lower doses are achieved in certain OARs with the 1F-PT plan compared to TriB-RT at cost of loss in PTV homogeneity and higher dose in other organs such as bladder and ipsilateral femoral head. The dose contributions of the TriB-RT plan for the three beam types are shown in figure 11 for a representative slice of the patient CT. It can be observed that the electron beams deliver a substantial contribution to the superficial part of the target volume, while the photon beams contribute mainly to the boundary 

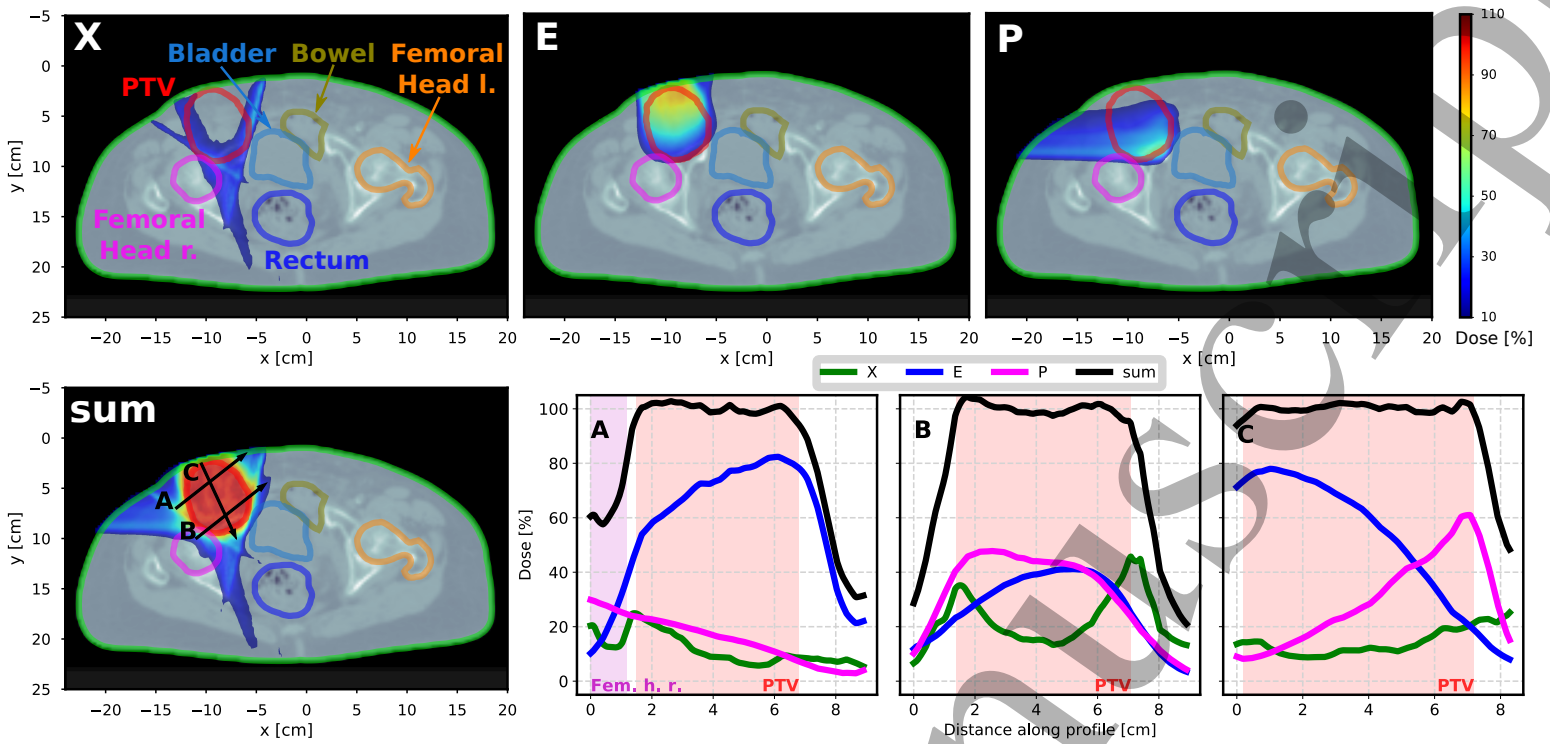

Figure 11: Top: Dose distributions by photon, electron and proton beams to the resulting TriB-RT plan for the pelvic case. Bottom: Total dose distribution and profiles indicating the respective contributions of the three particle types and corresponding dose profiles along the indicated arrows.

regions of the PTV. The contribution to the mean dose in the PTV are $18.8 \%, 47.9 \%$ and $33.3 \%$ for the photon, electron and proton beams, respectively.

\section{Discussion}

In this work, TriB-RT is presented - an MC-based TPP featuring the simultaneous optimization of photon, electron and proton beams. As a special case of this framework, MC-based IMPT optimization is enabled, which for an exemplary case shows at least equal plan quality compared to a corresponding proton plan generated in the Eclipse TPS. Other work has demonstrated the drawbacks of the TPS proton optimization due to the use of simplified dose calculation algorithms (Liu et al. 2019). With the presented in-house IMPT optimization, multi-field proton optimization based on MC beamlet calculation at custom calculation grid size is performed. Therefore, assuming no machine constraints, the resulting proton dose distributions are expected to be deliverable as such and no degradation due to simplified dose calculation has to be expected. Additional photon and electron fields are defined and all three particle 
1

2

3

4

5

6

7

8

9

types are simultaneously optimized in the process of generating a TriB-RT plan. It is noteworthy that although each proton field itself is able to cover the target volume on its own, a dosimetrically superior plan is found when allowing photon and electron beam contributions. For the investigated cases, photon beam contribution is most pronounced at the boundary of the target volume. This is consistent with the expected benefits of photon beams compared to proton beams regarding the steepness of the lateral penumbra and illustrates the potential of using photon beams to spare OARs in close proximity of the target volume, at cost of an increased low dose bath. Electron beam contribution is shown at the superficial parts of the respective target volumes. It is not intuitively obvious that electron beams are dosimetrically superior to proton beams for superficial targets. Both electron beams and proton spots have a larger penumbra compared to photon beams (Hogstrom et al. 2006; Safai et al. 2008), but have a finite range and are thus suited to reduce the low dose bath to normal tissue. This emphasizes the strength of the simultaneous optimization approach - the user bias is limited to choice of suitable beam directions and optimization objectives. All other aspects of the optimization such as relative contribution of the three particle types is incorporated in the optimization problem. The dosimetric benefit of TriB-RT compared to a proton only plan is substantially larger in the case, where a fixed proton beamline scenario with a single field is investigated. This indicates the potential of TriB-RT in a hospital environment, where an existing machine park of conventional linacs, which are able to deliver photon and electron beams, is extended with a cost-effective singleroom proton solution. The total cost of a TriB-RT treatment will therefore depend on the cost-efficiency of the individual treatment machines delivering the photon, electron and proton contribution and will depend on many parameters such as construction, personnel and maintenance cost (Goitein et al. 2003). For all investigated cases, TriB-RT provides a better solution to the optimization problem compared to IMPT plans. It should however be noted that the fluence map optimized TriB-RT plans provide a best-case solution to the optimization problem 


\section{$\operatorname{TriB}-R T$}

without the immediate demand for deliverability. Machine constraints could apply to the proton plans and the optimized photon and electron fluence maps would require either leaf sequencing of the pMLC (Gregoire et al. 2010) or a direct aperture optimization (DAO) approach (Shepard et al. 1999; Romeijn et al. 2005) to determine deliverable dose distributions. These aspects will be investigated in future work.

As with all treatment techniques in radiotherapy including TriB-RT, the final uncertainty of the dose distribution delivered to a patient depends on numerous factors. IMPT plans are known to be very sensitive to range and setup uncertainties, which could compromise treatment effectiveness (Lomax 2008a,b), whereas photon IMRT has been shown to be less sensitive to these uncertainties (Müller et al. 2015). Apart from the dosimetric benefits of TriB-RT for a static situation, treatment plans consisting of all three particle types might therefore be more robust against inter- and intrafraction uncertainties compared to IMPT alone. Renaud et al. 2019 have however concluded that for MBRT, robust optimization was required to maintain adequate target coverage under consideration of a set of positioning error scenarios. Given the nonuniformity of the dose distributions of the individual particle types in the presented TriB-RT plans, the assessment of such uncertainties is key to move towards clinical implementation. Robustness assessment and robust optimization of TriB-RT plans will therfore be investigated in future work.

\section{Conclusion}

TriB-RT, a novel treatment planning process with simultaneous optimization of modulated photon, electron and proton beams was successfully developed and studied for an academic and two clinically motivated cases. TriB-RT shows the potential for improved treatment quality and is especially promising for cost-effective singleroom proton solutions with a fixed beamline in combination with a conventional linac delivering the photon and electron fields. 
1

2

3

4

5

6

7

8

9

10

\section{REFERENCES}

\section{Acknowledgments}

This work was partially supported by Varian Medical Systems. Calculations were performed on UBELIX (http://www.id.unibe.ch/hpc), the HPC cluster at the University of Bern.

\section{References}

Bortfeld, T. (2006). "IMRT: a review and preview". In: Physics in Medicine \& Biology Vol. 51, No. 13, R363.

Broyden, C. G. (1970). "The convergence of a class of double-rank minimization algorithms: 2. The new algorithm". In: IMA journal of applied mathematics Vol. 6, No. 3, pp. 222-231.

Cho, B. (2018). "Intensity-modulated radiation therapy: a review/with a physics perspective". In: Radiation oncology journal Vol. 36, No. 1, p. 1 .

Deluca, P. M., A Wambersie, and G Whitemore (2007). Prescribing, Recording, and Reporting ProtonBeam Therapy (ICRU Report 78).

Ding, G. X., D. W. O. Rogers, and T. R. Mackie (1996). "Mean energy, energy-range relationships and depth-scaling factors for clinical electron beams", In: Medical Physics Vol. 23, No. 3, pp. 361-376.

Fix, M. K., P. Manser, D. Frei, W. Volken, R. Mini, and E. J. Born (2007). "An efficient framework for photon Monte Carlo treatment planning". In: Physics in Medicine \& Biology Vol. 52, No. 19, N425.

Fix, M. K. et al. (2013a). "Generalized eMC implementation for Monte Carlo dose calculation of electron beams from different machine types". In: Physics in Medicine 63 Biology Vol. 58, No. 9, pp. 2841-2859. ISSN: 00319155. DOI: 10.1088/0031-9155/58/9/2841. URL: http://www .ncbi.nlm. nih.gov/pubmed/23563175.

Fix, M. K., D. Frei, W. Volken, E. J. Born, D. M. Aebersold, and P. Manser (2013b). "Macro Monte Carlo for dose calculation of proton beams". In: Physics in Medicine and Biology Vol. 58, No. 7, pp. 2027-44. ISSN: 1361-6560. DOI: 10.1088/0031-9155/58/7/2027. URL: http://www.ncbi.nlm . nih.gov/pubmed/23458969.

Goitein, M and M Jermann (2003). "The relative costs of proton and X-ray radiation therapy". In: Clinical Oncology Vol. 15, No. 1, S37-S50.

Gregoire, V et al. (2010). ICRU Report No. 83 Prescribing, recording, and reporting photon-beam intensity-modulated radiation therapy (IMR). 


\section{REFERENCES}

Henzen, D et al. (2014a). "Beamlet based direct aperture optimization for MERT using a photon MLC." In: Medical Physics Vol. 41, No. 12, p. 121711. ISSN: 0094-2405. DOI: 10.1118/1.4901638. URL: http://www.ncbi.nlm.nih.gov/pubmed/25471958.

Henzen, D. et al. (2014b). "Forward treatment planning for modulated electron radiotherapy (MERT) employing Monte Carlo methods". In: Medical physics Vol. 41, No. 3, p. 31712.

Hogstrom, K. R. and P. R. Almond (2006). "Review of electron beam therapy physics". In? Physics in Medicine \& Biology Vol. 51, No. 13, R455.

Jeraj, R. and P. Keall (2000). "The effect of statistical uncertainty on inverse treatment planning based on Monte Carlo dose calculation". In: Physics in Medicine 63 Biology Vol. 45, No. 12, p. 3601.

Kawrakow, I. and M. Fippel (2000). "VMC++, a fast MC algorithm for radiation treatment planning". In: The Use of Computers in Radiation Therapy. Springer, pp. 126-128.

Kirova, Y. M. et al. (2007). "Postmastectomy electron beam chest wall irradiation in women with breast cancer: a clinical step toward conformal electron therapy". In: International Journal of Radiation Oncology* Biology* Physics Vol. 69, No. 4, pp. 1139-1144.

Kueng, R. et al. (2019). "Adaptive step size algorithm to increase efficiency of proton macro Monte Carlo dose calculation". In: Radiation oncology Vol. 14,No. 1, p. 165.

Liu, C. et al. (2019). "Treatment planning system (TPS) approximations matter - comparing intensitymodulated proton therapy (IMPT) plan quality and robustness between a commercial and an inhouse developed TPS for nonsmall cell lung cancer (NSCLC)". In: Medical physics Vol. 46, No. 11, pp. $4755-4762$.

Lomax, A. J. (2008a). "Intensity modulated proton therapy and its sensitivity to treatment uncertainties 1: the potential effects of calculational uncertainties". In: Physics in Medicine $\mathcal{G}$ Biology Vol. 53, No. 4, p. 1027.

- (2008b). "Intensity modulated proton therapy and its sensitivity to treatment uncertainties 2: the potential effects of inter-fraction and inter-field motions". In: Physics in Medicine 83 Biology Vol. 53 , No. 4, p. 1043.

Magaddino, V. et al. (2011). "Validation of the Swiss Monte Carlo Plan for a static and dynamic 6 MV photon beam". In: Zeitschrift fur Medizinische Physik Vol. 21, No. 2, pp. 124-134. Issn: 09393889. DOI: $10.1016 / j \cdot z e m e d i .2010 \cdot 10 \cdot 010$.

Míguez, C. et al. (2017). "Clinical implementation of combined modulated electron and photon beams with conventional MLC for accelerated partial breast irradiation". In: Radiotherapy and Oncology Vol. 124, No. 1, pp. 124-129.

Mohan, R. and D. Grosshans (2017). "Proton therapy-present and future". In: Advanced drug delivery reviews Vol. 109, pp. 26-44. 


\section{REFERENCES}

Mueller, S. et al. (2017). "Simultaneous optimization of photons and electrons for mixed beam radiotherapy". In: Physics in Medicine $\&$ Biology Vol. 62, No. 14, p. 5840. ISSN: 13616560. DOI: 10.1088/1361-6560/aa70c5. URL: http://stacks.iop.org/0031-9155/62/i=14/a=5840.

Mueller, S. et al. (2018a). "Electron beam collimation with a photon MLC for standard electron treatments". In: Physics in Medicine \&3 Biology Vol. 63, No. 2. ISSN: 13616560. Dor: 10.1088/13616560/aa9fb6.

Mueller, S. et al. (2018b). "Part 2: Dynamic mixed beam radiotherapy (DYMBER): Photon dynamic trajectories combined with modulated electron beams". In: Medical Physics Vol. 45, No. 9, pp. 42134226. ISSN: 00942405. DOI: $10.1002 / \mathrm{mp} .13085$.

Müller, B. S. et al. (2015). "Impact of interfractional changes in head and neck cancer patients on the delivered dose in intensity modulated radiotherapy with protons and photons". In: Physica Medica Vol. 31, No. 3, pp. 266-272.

Neuenschwander, H. and E. J. Born (1992). "A macro Monte Carlo method for electron beam dose calculations". In: Physics in Medicine $\& 3$ Biology Vol. 37, No. 1, pp. 107-125. ISSN: 00319155. DOI: 10.1088/0031-9155/37/1/007.

Neuenschwander, H., T. R. Mackie, and P. J. Reckwerdt/(1995). "MMC-a high-performance Monte Carlo code for electron beam treatment planning”. In: Physics in Medicine $\mathcal{E}$ Biology Vol. 40, No. 4, pp. 543-574. ISSN: 0031-9155. DOI: 10.1088/0031-9155/40/4/005.

Niemierko, A. (1999). "A generalized concept of equivalent uniform dose (EUD)". In: Med Phys Vol. 26, No. 6, p. 1100.

Nocedal, J. and S. Wright (1999). Numerical Optimization. Ed. by J. Nocedal and S. J. Wright. Springer Series in Operations Research and Financial Engineering. New York: Springer-Verlag. ISBN: 0-38798793-2. DOI: 10.1007/b98874. URL: http://link.springer.com/10.1007/b98874.

Otto, K. (2008). "Volumetric modulated arc therapy: IMRT in a single gantry arc". In: Medical physics Vol. 35, No. 1, pp. 310-317.

Paganetti, H. (2016). Proton Béam Therapy. 2399-2891. Philadelphia: IOP Publishing. IsBn: 978-07503-1370-4. DOI: 10.1088/978-0-7503-1370-4. URL: https : / www . uclh . nhs . uk/aboutus / NewDev/NCF / PBT / Pages / Home . aspxhttp : / / iopscience . iop . org/book/978-0-7503-13704http://dx.doi.org/10.1088/978-0-7503-1370-4.

Renaud, M.-A., M. Serban, and J. Seuntjens (2017). "On mixed electron-photon radiation therapy optimization using the column generation approach". In: Medical Physics Vol. 44, No. 8, pp. 42874298. (2019). "Robust mixed electron-photon radiation therapy optimization". In: Medical physics Vol. 46, No. 3, pp. 1384-1396. 


\section{REFERENCES}

Romeijn, H. E., R. K. Ahuja, J. F. Dempsey, and A. Kumar (2005). "A column generation approach to radiation therapy treatment planning using aperture modulation". In: SIAM Journal on Optimization Vol. 15, No. 3, pp. 838-862.

Safai, S., T. Bortfeld, and M. Engelsman (2008). "Comparison between the lateral penumbra of a collimated double-scattered beam and uncollimated scanning beam in proton radiotherapy". In: Physics in Medicine ES Biology Vol. 53, No. 6, p. 1729.

Schultheiss, T. E. (2017). "The Impact of IGRT on Normal Tissue Toxicity" In: Advances in Radiation Oncology. Springer, pp. 145-154.

Shepard, D. M., M. C. Ferris, G. H. Olivera, and T. R. Mackie (1999). "Optimizing the delivery of radiation therapy to cancer patients". In: Siam Review Vol. 41, No. 4, pp. 721-744.

Unkelbach, J., M. Bangert, K. D. A. Bernstein, N. Andratschke, and M. Guckenberger (2018). "Optimization of combined proton-photon treatments". In: Radiotherapy and Oncology Vol. 128, No. 1, pp. 133-138.

Varian Medical Systems (2014). "Eclipse Photon and Electron Reference Guide". In:

Walters, B. R. B., I Kawrakow, and D. W. O. Rogers (2002). "History by history statistical estimators in the BEAM code system". In: Medical physics Vol. 29, No. 12, pp. 2745-2752.

$\mathrm{Wu}, \mathrm{Q}$. and R. Mohan (2000). "Algorithms and functionality of an intensity modulated radiotherapy optimization system". In: Medical Physics Vol. 27, No. 4, pp. 701-711.

Yu, C. X. (1995). "Intensity-modulated arc therapy with dynamic multileaf collimation: an alternative to tomotherapy". In: Physics in Medicine \& Biology Vol. 40, No. 9, p. 1435. 
Appendix A. MC re-calculation and IMPT optimization for water equivalent situation
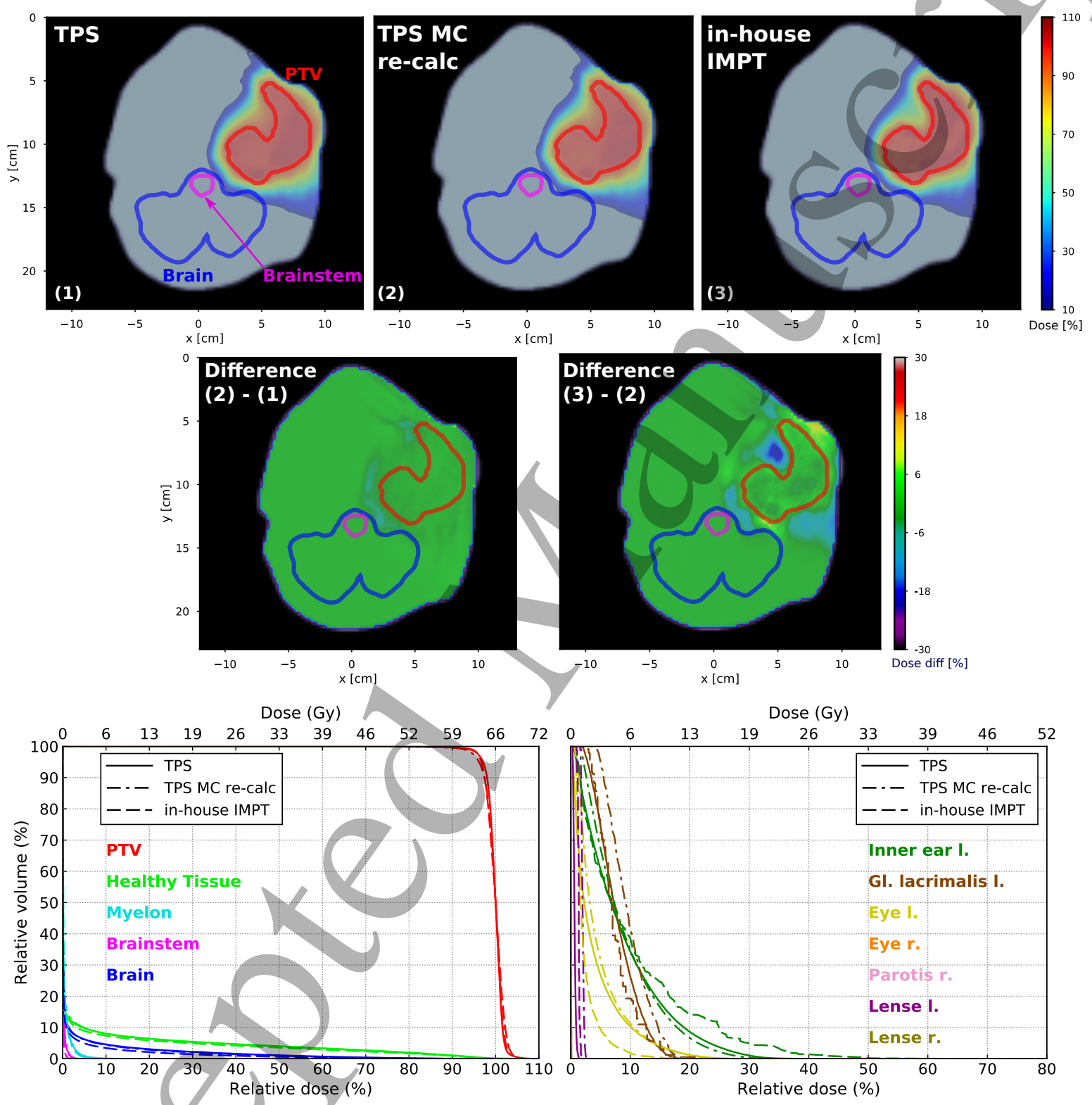

Figure A1: Top: Dose distributions of the TPS optimized proton plan (left), the same plan recalculated with $M C$ (center), and the in-house optimized IMPT plan (right) for a representative slice of the planning CT, with PTV contour indicated in red. Middle: Relative dose difference (\% of prescription dose) between the re-calculated and original TPS plan (left) and between in-house IMPT and MC re-calculated TPS plan (right). Bottom: DVHs comparing the resulting TPS optimized proton plan (solid), re-calculated TPS plan (dash-dot) and in-house optimized IMPT plan (dashed) for the water equivalent head and neck case. 